\title{
A de novo virus-like topology for synthetic virions
}

Article

Accepted Version

Noble, J. E., De Santis, E. ., Ravi, J., Lamarre, B., Castelletto, V., Ray, S. and Ryadnov, M. G. (2016) A de novo virus-like topology for synthetic virions. Journal of the American Chemical Society, 138 (37). pp. 12202-12210. ISSN 00027863 doi: https://doi.org/10.1021/jacs.6b05751 Available at https://centaur.reading.ac.uk/67291/

It is advisable to refer to the publisher's version if you intend to cite from the work. See Guidance on citing.

To link to this article DOI: http://dx.doi.org/10.1021/jacs.6b05751

Publisher: American Chemical Society

All outputs in CentAUR are protected by Intellectual Property Rights law, including copyright law. Copyright and IPR is retained by the creators or other copyright holders. Terms and conditions for use of this material are defined in the End User Agreement.

\section{www.reading.ac.uk/centaur}

\section{CentAUR}

Central Archive at the University of Reading 
Reading's research outputs online 


\title{
A de novo ultra-small, anionic and size-adaptable
}

\section{topology of virus assembly for functional gene}

\section{delivery}

James E Noble, ${ }^{1}$ Emiliana De Santis, ${ }^{1}$ Jascindra Ravi, ${ }^{1}$ Baptiste Lamarre, ${ }^{1}$ Valeria Castelletto, ${ }^{2}$

Santanu Ray, Maxim G Ryadnov ${ }^{1, *}$

\author{
${ }^{1}$ National Physical Laboratory, Hampton Road, Teddington, TW11 0LW, UK \\ ${ }^{2}$ Department of Chemistry, University of Reading, RG6 6AD, UK \\ ${ }^{3}$ SET, University of Brighton, BN2 4GJ, UK
}

Corresponding author:

Dr Maxim G Ryadnov

National Physical Laboratory,

Hampton Road, Teddington, TW11 0LW, UK

Fax: (+44) 2086140573

Tel: (+44) 2089436078

max.ryadnov@npl.co.uk 


\begin{abstract}
A de novo ultra-small topology of viral assembly is reported. The design is a trifaceted coiled-coil peptide helix, which self-assembles into monodisperse, anionic virions able to encapsulate and transfer both RNA and DNA into human cells. Unlike existing artificial systems, the virions share the same physical characteristics of viruses being anionic, non-aggregating, abundant, hollow and uniform in size, while effectively mediating gene silencing and transgene expression. These are the smallest virions reported to date with the ability to adapt and transfer small and large nucleic acids thus offering a promising solution for engineering bespoke artificial viruses with desired functions.
\end{abstract}

Keywords: self-assembly $\bullet$ artificial virus $\bullet$ gene silencing $\bullet$ de novo design $\bullet$ nanoshells

Viruses are hollow nano-shells encasing nucleic acids (NAs). ${ }^{1}$ The shells range from $17 \mathrm{~nm}$ (porcine cicrovirus) ${ }^{2}$ to $1 \mu \mathrm{m}$ (pandoravirus), ${ }^{3}$ and all self-assemble from individual protein subunits. ${ }^{1}$ The subunits are conserved protein folds programmed to reproduce the viral assembly, which inspires the search for synthetic analogues. ${ }^{4}$ Different approaches and chemistries are being proposed, though a primary emphasis is often placed on applications rather than the structural cooperativity of viral designs. ${ }^{5-10}$ As a result, amorphous, aggregating and polydisperse structures are common products. ${ }^{10}$ Recent peptide designs offer promising solutions. ${ }^{11-13}$ However, these lack the morphological uniformity of viruses, while their ability to infect and mediate genetic processes remains to be demonstrated. Here we introduce a de novo self-assembly topology which mitigates these shortcomings and provides a biologically functional mimetic of the viral assembly.

The virus architecture adopts an $n$-fold rotational symmetry, where $n$ can be 3 or 5 or both. ${ }^{1}$ Therefore, adapting a virus-like architecture requires a folding unit able to support at least a 
symmetric $3 \mathrm{D}\left(C_{3}\right)$ assembly. ${ }^{14} \mathrm{~A}$ chemical approach to achieve this using relatively short peptide sequences, as opposed to fully folded proteins, ${ }^{15}$ is to template the assembly of a folding unit on a macromolecular framework that can support the same properties of symmetry, monodispersity and hierarchical assembly. In this light, the dendrimer architecture lends itself as an intrinsic template for $C_{3}$-assembly. ${ }^{16,17}$ Specifically, our design converts dendrimer branching cells, which are covalent structures, into supramolecular focal points (Fig 1A). ${ }^{18}$ Introduced as modular nanosphere clusters to benefit from monodispersity and dimensional scaling of globular proteins, self-assembling or tecto-

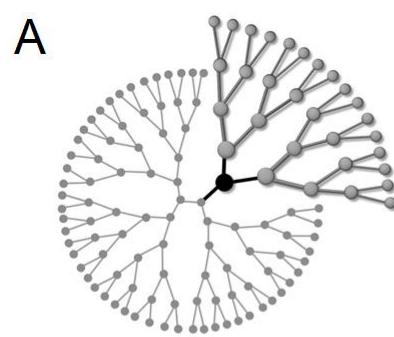

dendrimer architecture

C

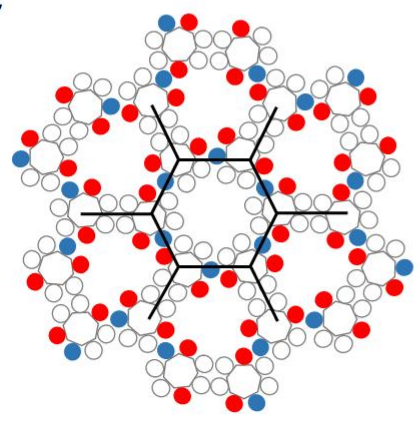

tecto-dendrimer unit
B gabcdefgabcdefgabcdefgabcde CGG-EIARLEQEIARLEQEIARLEYEIARLE-am

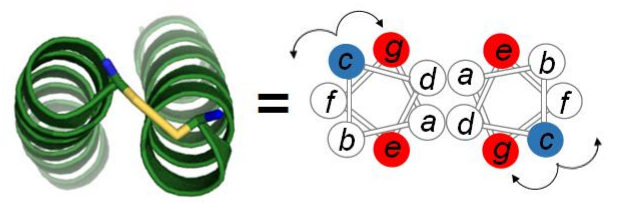

self-assembling subunit

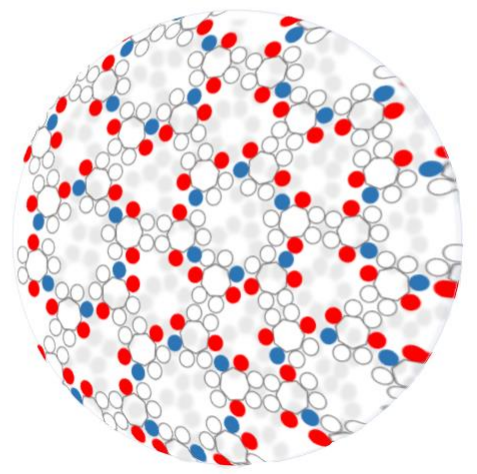

assembled virion

dendrimers are analogous to viral particles in that both assemble from subunits around an inner focal point or cavity. ${ }^{1,19,20}$ Thus, utilizing the dendrimer topology in a virus-like design serves the same benefits.

Figure 1. Tecto-dendrimeric virus-like (TecVir) design. (A). Schematic representation of the dendrimer architecture highlighting a branching cell (bold black) in a branch zoomed in for clarity. (B) TecVir sequence (top view) and its coiled-coil subunit (lower, left) configured into helical wheels with 3.5 residues per turn (lower, right). The subunit (PDB entry 4DMD rendered by PyMOL) 
highlights a cysteine bridge (yellow). The sequences show heptad repeats of canonical coiled coils, designated gabcdef. Residues at $a, d$ sites form the hydrophobic interface, and are isoleucine and leucine, respectively, favoring dimer formation. Residues at $e$ and $g$ are anionic glutamates (red) that interact with cationic arginines (blue) at $c$ forming intra-helical $(g-c)$ interactions in the same dimer and inter-helical $\left(c-e^{\prime}\right)$ interactions between dimers. The arrows indicate intra- and inter-helical interactions. (C) Each helix is a branching cell interacting with three other helices giving rise to a branching network (left) which closes into a shell (right).

With this in mind, we designed a tecto-dendrimeric virus-like shell (TecVir). The design is based on a self-complementary coiled-coil subunit having three interfacial facets. The subunit is an $\alpha$-helix which self-pairs via a hydrophobic interface typical of a coiled coil dimer (Fig 1B). ${ }^{21}$ In canonical coiled coils each helix has one polar face and one hydrophobic face, which interact with counterpart helices, one in dimers and two in higher oligomers. ${ }^{22}$ By contrast, in this design each individual helix has one hydrophobic interface and two polar facets that are arranged to favor interactions with other three, but identical, neighbors (Fig 1B\&C). ${ }^{23}$ Thus, each helix can be viewed as a branching cell in a continuous branching network whose propagation axis runs perpendicular to that of individual helices. All helices pack laterally, which facilitates the closing of an increasingly curved network into a shell. ${ }^{14,23,24}$ To support this transition the peptide was extended at the N-terminus with a cysteinecontaining motif CGG (Fig 1 and Table s1 in Supporting Information). The tight inter-helix packing is expected to promote proximity-driven oxidation of cysteine residues cementing the structure. ${ }^{25}$ Without this cementing effect the $C_{3}$-symmetric networks would not be complete, resulting in more anisotropic assemblies such as nanofibres. ${ }^{13}$

Consistent with this, cryogenic transmission electron microscopy (cryo-TEM) revealed abundant and monodisperse $(12 \pm 1.5 \mathrm{~nm})$ spherical shells for TecVir at micromolar concentrations (Fig 2). Small angle X-ray scattering (SAXS) was performed to probe the assembly directly in solution. Fitting of the SAXS data biased for a co-existence of polydisperse spherical shells with homogeneous 
cylinders in solution, revealed a dominating external radius of a spherical shell $\left(R_{l}\right)$ centered at $6.5 \pm$ $2.3 \mathrm{~nm}$ and a wall thickness $\left(t h_{1}\right)$ of $4 \mathrm{~nm}$ together with an additional $R_{2}$ of $11.6 \pm 5 \mathrm{~nm}$ with a $t h_{2}$ of $5.8 \mathrm{~nm}$ (Fig 3A, B, Table s2). ${ }^{26}$ The values for the two spherical shells overlapped and were in good agreement with the cryo-TEM data confirming that the vesicles were largely monodisperse and hollow. ${ }^{11,27}$ The wall thickness matches the span of the coiled-coil building blocks (4-4.4 nm), which is indicative of peptides orientating parallel to the axis of rotation in the shells as shown in Figure 1C. The fitting of the SAXS profile also incorporates a contribution from rod-like $4 \times 2 \mathrm{~nm}$ cylinders. This finding agrees with the dimensions of individual coiled-coil subunits $(4 \times 2 \mathrm{~nm})^{22}$ that remained in equilibrium with the assembled shells (Table s2), which is characteristic of cooperative and reversible assemblies. $^{28,29}$
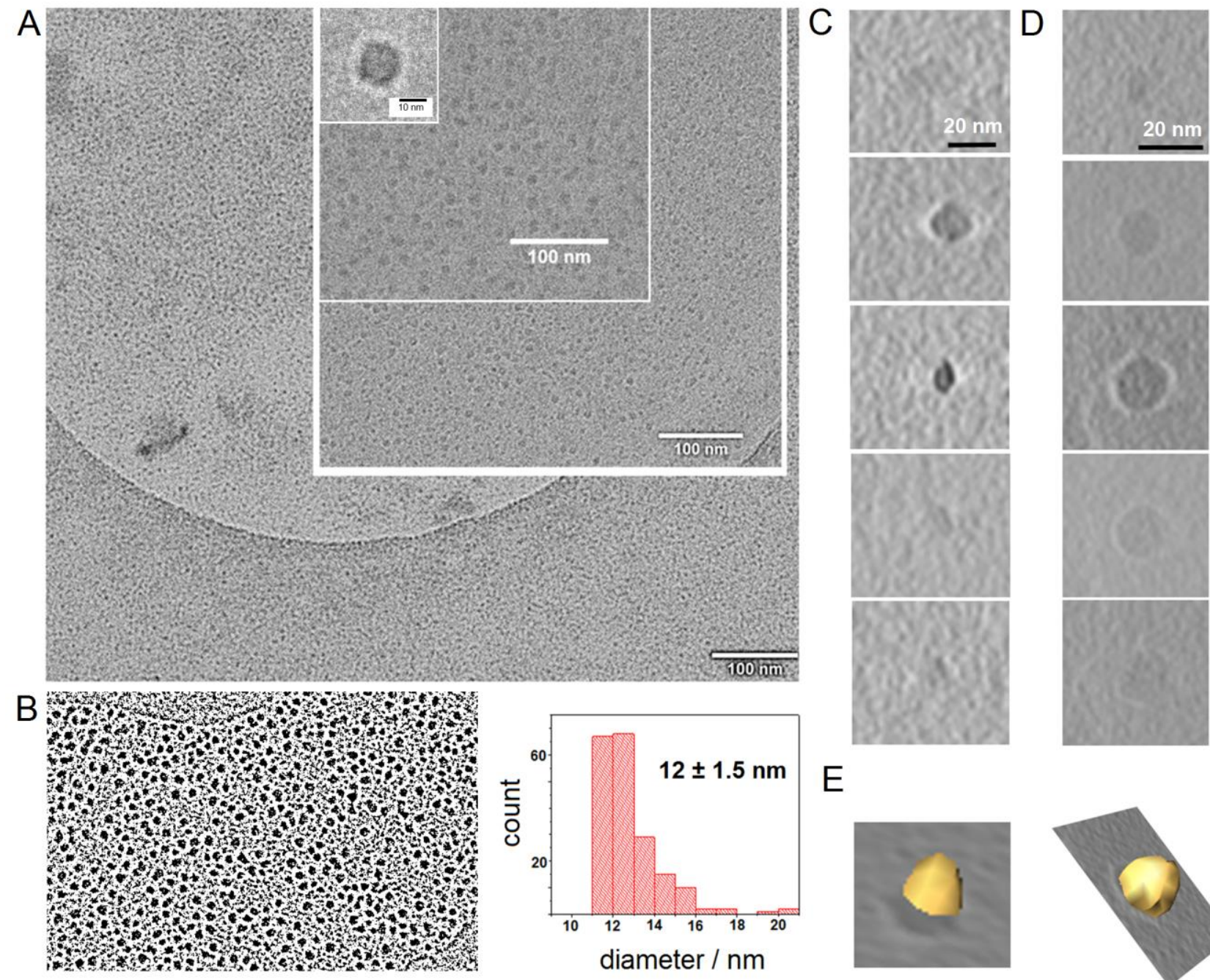

$E$
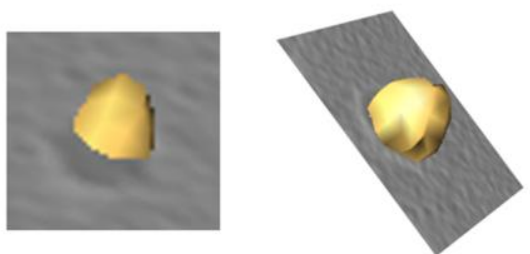
Figure 2. TecVir assembly. (A) Cryo-electron micrographs of assembled shells, and (B) a representative contrast-inverted image analysis (right). (C, D) Cryo-electron tomography (z-planes) for TecVir (C) and for TecVir assembled with siRNA (D) at the N/P molar ratio of 1/76 at $100 \mu \mathrm{M}$ peptide, and (E) corresponding iso-surface reconstructions.

Circular dichroism (CD) spectroscopy confirmed appreciable helix formation for the assembly while spectral $\Delta \varepsilon_{222} / \Delta \varepsilon_{208}$ ratios were $\geq 1$, as expected for helical oligomers as opposed to monomeric helices (Fig 3C, s1A). ${ }^{30}$ The assembly proved to be highly cooperative as gauged by sigmoidal unfolding curves with a single transition midpoint $\left(T_{M}\right)$ of $\sim 55^{\circ} \mathrm{C}$ (Fig s1B). Such a stable structure was found to be fully reversible with spectra recorded before and after the melt being nearly identical. During thermal denaturation $\left(20-90^{\circ} \mathrm{C}\right)$ signal intensity at $202 \mathrm{~nm}$ remained the same providing a clear isodichroic point indicating a two-state transition between helical and unfolded forms (Fig s1A). In contrast, an Arg $\rightarrow$ Ala mutant of the peptide, TecVir ${ }^{\text {ala }}$, designed as a negative control incapable of self-assembly did not fold or assemble (Fig 3C, s1A and Table s1). TecVir with the cysteine residue capped via thioalkylation, $\mathrm{TecVir}^{\mathrm{cap}}$, did fold, as expected, but the first derivatives of its unfolding curves suggested overlapping conformer populations with a predominant $T_{M}$ of $65^{\circ} \mathrm{C}$ (Fig s1C).

In accord with the data, microscopy revealed co-existent morphological forms: polydisperse particles and more anisotropic assemblies, extended nanofibers (Fig s2). This finding is consistent with the stabilisation effect of the cysteine residues on the assembly of the coiled-coil networks and with the formation of disulphide bridges. Indeed, the comparative quantification of free thiols in TecVir by the Ellman's test gave up to $100 \%$ reductions in the concentration of free thiols in the assembly confirming that cysteine residues were oxidised. Combined the results indicate that TecVir folds into a hierarchical network supporting the formation of homogeneous nanoscale shells. 

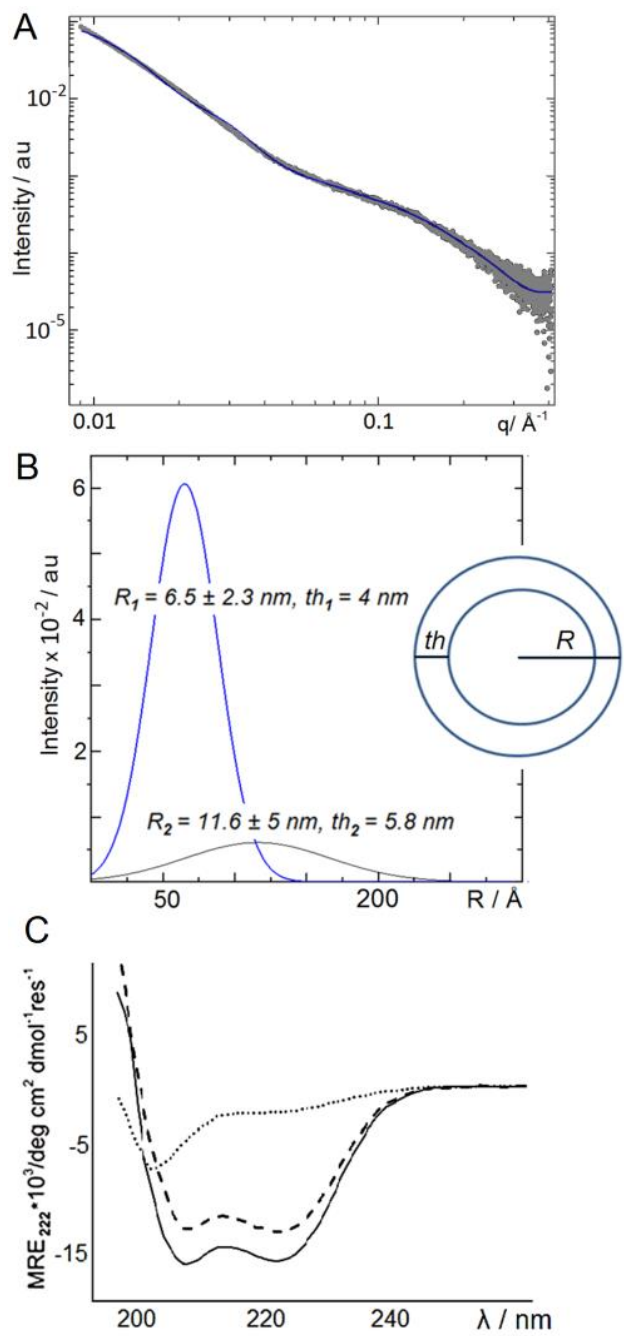

Figure 3. TecVir folding. (A) SAXS profile (grey dots) for TecVir shells and (B) Gaussian distributions corresponding to the radii $(R)$ and wall thicknesses $(t h)$ of two hollow spheres (blue and grey). The fitting curve (dark blue) in A incorporates contributions from the two spheres and a $4 \times 2$ nm cylinder (coiled-coil dimer). (C) CD spectra for TecVir (solid line), TecVir ${ }^{\text {cap }}$ (dashed line) and TecVir $^{\text {ala }}$ (dotted line): $100 \mu \mathrm{M}$ peptide, $\mathrm{pH}$ 7.4, $10 \mathrm{mM}$ MOPS, $1 \mathrm{mM}$ TCEP (TecVir), $20^{\circ} \mathrm{C}$.

To function, viral mimetics must infect cells. To gain an insight into its biological relevance, TecVir was probed for gene delivery. With the net charge of -3 (Table s1), TecVir assembles into anionic shells ( $\zeta$-potential of $-42.4 \pm 0.73$, see Supporting Information), which, unlike for most gene-delivery agents, disfavours purely electrostatic complexation with negatively charged NAs. Therefore, encapsulation is necessary. The free $\mathrm{N}$-terminus of the peptide contributes a positive charge for the 
coiled-coil units to assemble around NAs, which was envisaged to require low NA-peptide (N/P) ratios. Indeed, TecVir co-assembled with small interfering RNA (siRNA) at N/P molar ratios of 1/40-1/100 promoted appreciable inhibition of targeted gene expression (Fig 4A). At these ratios the size of TecVir assemblies increased giving a mean diameter of $26.5 \pm 8.7 \mathrm{~nm}$ (Fig $2 \mathrm{D}$, E and Fig s3), suggesting the encapsulation of siRNA in TecVir shells. Given that the siRNA duplexes used in the study are $~ 7.5 \mathrm{~nm}$ in length (23 bases) and $2 \mathrm{~nm}$ (B form) in diameter, ${ }^{31}$ an individual TecVir shell has to double in diameter $(4 \mathrm{~nm})$ in order to accommodate one or two siRNA duplexes, which appeared to be consistent with the overall size increases.

\section{A SIRNA}

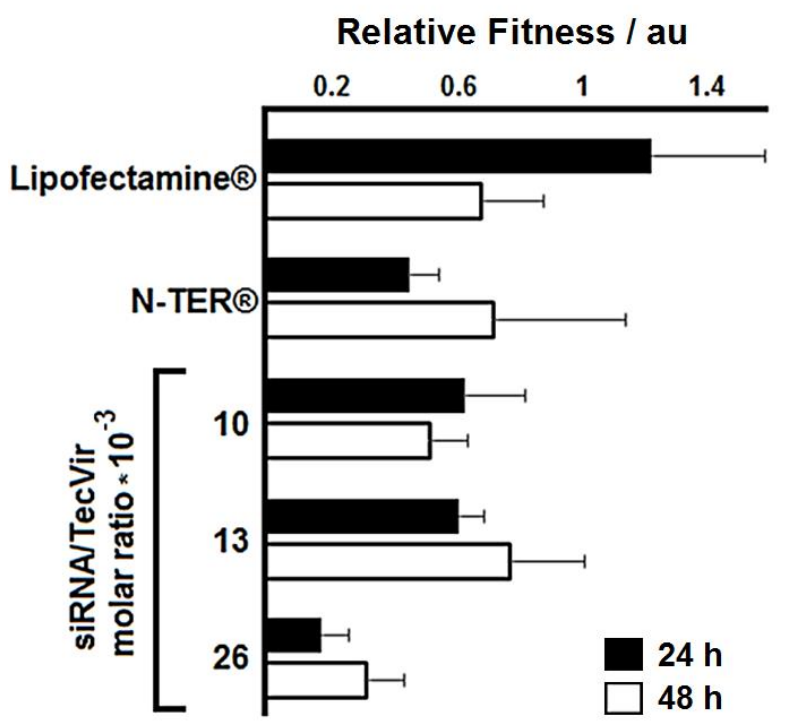

\section{B DNA}

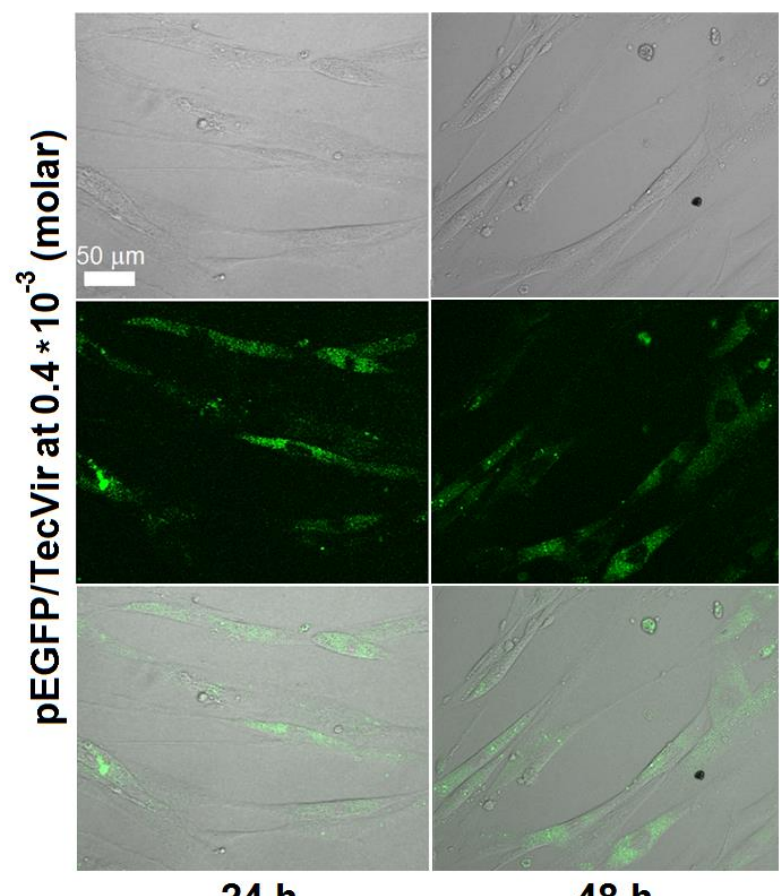

Figure 4. TecVir biological activity. TecVir-promoted gene transfer, expression (DNA) and silencing (siRNA). (A) Knockdown fitness of TecVir and commercial Lipofectamine ${ }^{\circledR}$ RNAiMAX and N-TER ${ }^{\circledR}$ (positive controls) normalized against siRNA alone (negative control) and the total counts of viable cells at different siRNA/TecVir molar ratios at $37 \mathrm{nM}$ siRNA. (B) Widefield (left), fluorescence (middle) and combined (right) micrographs of human dermal fibroblasts transfected with plasmid DNA encoding for green fluorescent protein (green). GFP expression measured after 24 and 48 hours is shown. 
These siRNA-containing assemblies efficiently transfected cells and mediated gene knockdown which was monitored by reverse transcription polymerase chain reaction (RT-PCR) at the mRNA level using HeLa cells with two housekeeping genes, ACTB ( $\beta$-actin, targeted) and GAPDH (reference). ${ }^{32}$ The silencing of $\beta$-actin mRNA was detected at $24 \pm 2$ hours and $48 \pm 2$ hours posttransfection, with knockdown efficiency being comparable to that of commercial peptide (N-Ter®) and liposomal (Lipofectamine® RNAiMAX) reagents (Fig 4A). Higher N/P ratios gave negligible knockdown levels, while no obvious increases were observed for lower N/P ratios. By marked contrast, siRNA/TecVir complexes that were attempted by adding siRNA into pre-assembled TecVir shells failed to yield reproducible knockdown, confirming that co-assembly is necessary for encapsulation.

Monodispersity for viruses is a constraint ensuring the encapsulation of viral genes. Viral capsids tend to resist increases in cargo sizes, but may adapt by increasing the outer diameters through capsid packing defects. ${ }^{33}$ The TecVir shells are uniform and homogeneous in size, and are also structurally adaptable for encapsulating a larger genetic cargo. To test this, TecVir was co-assembled with a large plasmid DNA encoding for the enhanced green fluorescent protein (pEGFP) and the obtained vehicles were used to transfect primary cells, human dermal fibroblasts (Fig 4B). Strikingly, lower N/P molar ratios, as expected for larger NAs, gave transfection efficiencies comparable with those of a liposomal reagent Lipofectamine® 2000, while EGFP expression peaked at $80 \%$ within 48 hours of incubation for both reagents with no apparent changes in cell morphology (Fig 4B and s4A). Biological activity (EGFP expression and mRNA silencing) was not associated with cytotoxic effects. In addition to unaltered cell morphology during transfections (Fig 4B), cell viability AlamarBlue ${ }^{\circledR}$ assays, which provide quantitative indicators of metabolically active cells, revealed that TecVir-treated cells remained viable over the entire transfection periods (up to 48 hours) (Fig s4B). 
In summary, by combining the principles of chemical and de novo protein design we have engineered self-assembling peptide shells as structural and functional mimetics of viruses. These are compact and small nanoshells that can adapt to encapsulate both RNA and DNA. Similar to viruses, these shells self-assemble from individual subunits and promote functional gene transfer into live cells. Unlike viral subunits, which are large and conserved proteins, TecVir is built of much smaller peptides. This is beneficial for the development of bespoke artificial viruses because peptides are more synthetically accessible, their chemistry allows substantial orthogonality for topology and functionalization, while peptide folding-assembly pathways can be predictably engineered using basic protein folding motifs. In functional terms, TecVir forms virus-like nanoshells with pI (4.5) that falls within the range of viral isoelectric points of $3.5-7 .{ }^{34}$ This property holds a particular promise for engineering small anionic virions with the ability to cross blood brain barrier or circumvent rapid clearance from circulation and cytotoxicity, which for cationic reagents remain major obstacles towards efficient gene therapy approaches. ${ }^{35}$

\section{REFERENCES}

1. Perlmutter, J. D.; Hagan, M. F. Annu Rev Phys Chem., 2015, 66, 217.

2. Yin, S.; Sun, S.; Yang, S.; Shang, Y.; Cai, X.; Liu, X. Virol J., 2010, 7, 166.

3. Philippe, N. et al. Science, 2013, 341, 281.

4. Mastrobattista, E.; van der Aa, M.A.; Hennink, W. E.; Crommelin, D. J. Nat Rev Drug Discov., 2006, 5, 115.

5. Marsden, H. R.; Korobko, A. V.; van Leeuwen, E. N. M.; Pouget, E. M.; Veen, S. J. Sommerdijk, N. A. J. M.; Kros, A. J Am Chem Soc., 2008, 130, 9386.

6. Ni, R.; Chau, Y. J Am. Chem. Soc., 2014, 136, 17902.

7. Olson, A. J.; Hu, Y. H. E.; Keinan, E. Proc. Natl Acad. Sci. USA, 2007, 104, 20731.

8. Nakai, T.; Kanamori, T.; Sando, S.; Aoyama, Y. J Am. Chem. Soc., 2003, 125, 8465.

9. Holowka, E. P.; Sun, V. Z.; Kamei, D. T.; Deming, T. J. Nat Mater., 2007, 6, 52. 
10. Lamarre, B.; Ryadnov, M. G. Macromol. Biosci., 2011, 11, 503.

11. Matsuura, K.; Watanabe, K.; Matsuzaki, T.; Sakuri, K.; Kimizuka, N. Angew. Chem. Int. Ed., 2010, 49, 9662.

12. Tarasov, S. G.; Gaponenko, V.; Howard, O. M.; Chen, Y.; Oppenheim, J. J.; Dyba, M. A.; Subramaniam, S.; Lee, Y.; Michejda, C.; Tarasova, N. I. Proc. Natl Acad. Sci. USA, 2011, 108, 9798.

13. Matsuura, K.; Murasato, K.; Kimizuka, N. J Am Chem. Soc., 2005, 127, 10148.

14. Castelletto, V. et al. Chem. Sci., 2016, 7, 1707.

15. King, N. P. et al. Nature, 2014, 510, 103.

16. Frechet, J. M. J. Proc Natl Acad. Sci. USA., 2002, 99, 4782.

17. Lin, B. F. et al. Nano Lett., 2011, 11, 3946.

18. S. Uppuluri, S.; Swanson, D. R.; Piehler, L. T.; Li, J.; Hagnauer, G. L.; Tomalia, D. A. Adv. Mater, 2000, 11, 796.

19. Tomalia, D. A.; Brothers, H. M.; Piehler, L. T.; Durst, H. D.; Swanson, D. R. Proc Natl Acad. Sci. USA., 2002, 99, 5081.

20. Wong, P. T.; Tang, K.; Coulter, A.; Tang, S.; Baker, J. R.; Choi, S. K. Biomacromolecules, 2014, 15, 4134.

21. Apostolovic, B.; Danial, M.; Klok, H.-A. Chem. Soc. Rev., 2010, 39, 3541.

22. Lupas, A. Trends Biochem. Sci., 1996, 21, 375.

23. Ryadnov, M. G. Angew. Chem. Int. Ed., 2007, 46, 969.

24. Fletcher J. M. et al. Science, 2013, 340, 595.

25. Ryadnov, M. G.; Woolfson, D. N. J Am Chem. Soc., 2007, 129, 14074.

26. Breßler, I.; Kohlbrecher, J.; Thünemann, A. F. J Appl Cryst, 2015, 48, 1587.

27. Hirai, M.; Iwase, H.; Hayakawa, T.; Koizumi, M.; Takahashi, H. Biophys. J. 2003, 85, 1600.

28. Zlotnick, A. J Mol. Biol., 1994, 241, 59. 
29. Hernandez-Garcia, A. et al. Nat Nanotechnol., 2014, 9, 698.

30. Kelly, S. M.; Jess, T. J.; Price, N. C. Biochim. Biophys. Acta, 2005, 1751, 119.

31. Schroeder, A.; Levins, C. G.; Cortez, C.; Langer, R.; Anderson, D. G. J Intern Med, 2010, $267,9$.

32. Bustin, S. A. et al. Clin. Chem., 2009, 55, 611.

33. He, L.; Porterfield, Z.; van der Schoot, P.; Zlotnick, A.; Dragnea, B. ACS Nano, 2013, 7, 8447.

34. Michen, B. T.; Graule T. J Appl Microbiol. 2010, 109, 388.

35. Tagalakis, A. D. et al. Biomaterials, 2014, 35, 8406.

Supporting information contains additional scheme, tables and figures

\section{Acknowledgements}

We acknowledge funding from the UK's Department of Business, Innovation and Skills. We thank J. Mantel (University of Bristol) for support with the electron microscopy experiments, I. W. Hamley for access to his B21 beamtime (Ref. 12321-1) awarded at Diamond Light Source and K. Inoue for assistance during the beamtime. 